Proceedings of the Edinburgh Mathematical Society (2004) 47, 561-572 (C)

DOI:10.1017/S0013091503000956 Printed in the United Kingdom

\title{
CONVOLUTION SUMS INVOLVING THE DIVISOR FUNCTION
}

\author{
NATHALIE CHENG AND KENNETH S. WILLIAMS \\ Centre for Research in Algebra and Number Theory, \\ School of Mathematics and Statistics, \\ Carleton University, Ottawa, ON K1S 5B6, \\ Canada (williams@math.carleton.ca)
}

(Received 13 November 2003)

Abstract The series

$$
\begin{aligned}
& L_{r, 4}(q)=\sum_{n=0}^{\infty} \sigma(4 n+r) q^{4 n+r}, \quad r=0,1,2,3, \\
& M_{r, 4}(q)=\sum_{n=0}^{\infty} \sigma_{3}(4 n+r) q^{4 n+r}, \quad r=0,1,2,3, \\
& N_{r, 4}(q)=\sum_{n=0}^{\infty} \sigma_{5}(4 n+r) q^{4 n+r}, \quad r=0,1,2,3,
\end{aligned}
$$

are evaluated and used to prove convolution formulae such as

$$
\sum_{m \leqslant n} \sigma(4 m-3) \sigma(4 n-(4 m-3))=4 \sigma_{3}(n)-4 \sigma_{3}\left(\frac{1}{2} n\right) .
$$

Keywords: divisor function; convolution sum; arithmetic identity

2000 Mathematics subject classification: Primary 11A25

\section{Introduction}

For $f, n \in \mathbb{N}$, we let $\sigma_{f}(n)$ denote the sum of the $f$ th powers of the positive divisors of $n$ :

$$
\sigma_{f}(n)=\sum_{d \mid n} d^{f}
$$

If $n \notin \mathbb{N}$, we set $\sigma_{f}(n)=0$. We set $\sigma_{1}(n)=\sigma(n)$.

In 1916 Ramanujan (see [7] and [9, pp. 136-162]) introduced the three functions $L(q)$, $M(q)$ and $N(q)$ defined for $q \in \mathbb{C}$ with $|q|<1$ by

$$
L(q)=1-24 \sum_{n=1}^{\infty} \sigma(n) q^{n}
$$




$$
\begin{aligned}
& M(q)=1+240 \sum_{n=1}^{\infty} \sigma_{3}(n) q^{n}, \\
& N(q)=1-504 \sum_{n=1}^{\infty} \sigma_{5}(n) q^{n} .
\end{aligned}
$$

It is known that the series $L(q), M(q)$ and $N(q)$ are algebraically independent [10, p. 69]. Ramanujan (see [7] and [9, pp. 136-162]) proved (among many others) the two formulae

$$
L^{2}(q)=M(q)+12 q \frac{\mathrm{d} L}{\mathrm{~d} q}
$$

and

$$
L(q) M(q)=N(q)+3 q \frac{\mathrm{d} M}{\mathrm{~d} q},
$$

from which follow, by equating the coefficients of $q^{n}$ on both sides, the arithmetic identities

$$
\sum_{m<n} \sigma(m) \sigma(n-m)=\frac{5}{12} \sigma_{3}(n)+\frac{1}{12} \sigma(n)-\frac{1}{2} n \sigma(n)
$$

and

$$
\sum_{m<n} \sigma(m) \sigma_{3}(n-m)=\frac{7}{80} \sigma_{5}(n)-\frac{1}{8} n \sigma_{3}(n)+\frac{1}{24} \sigma_{3}(n)-\frac{1}{240} \sigma(n)
$$

In all Ramanujan obtained nine identities of the type (1.6) and (1.7). For the history of such formulae see [4].

In this paper we consider the related series

$$
\begin{array}{ll}
L_{r, 4}(q) & =\sum_{n=0}^{\infty} \sigma(4 n+r) q^{4 n+r}, \quad r=0,1,2,3, \\
M_{r, 4}(q) & =\sum_{n=0}^{\infty} \sigma_{3}(4 n+r) q^{4 n+r}, \quad r=0,1,2,3 \\
N_{r, 4}(q) & =\sum_{n=0}^{\infty} \sigma_{5}(4 n+r) q^{4 n+r}, \quad r=0,1,2,3 .
\end{array}
$$

In $\S 2$ we obtain formulae for $L_{r, 4}(q), M_{r, 4}(q)$ and $N_{r, 4}(q)(r=0,1,2,3)$ similar to those given by Ramanujan (see [7] and [9, pp. 136-162]) for $L(q), M(q)$ and $N(q)$ (see Theorem 2.1). In $\S 3$ we use these formulae to determine which products $L_{r, 4}(q) L_{s, 4}(q)$ and $L_{r, 4}(q) M_{s, 4}(q)(0 \leqslant r \leqslant s \leqslant 3)$ can be expressed in terms of the functions $L, M$ and $N$ and their derivatives (see Theorem 3.1). As a consequence of these identities we obtain, in $\S 4$, a number of arithmetic identities analogous to (1.6) and (1.7) (see Theorem 4.1). In $\S 5$ we prove the formulae

$$
L(q) L\left(q^{2}\right)=\frac{1}{5}\left(M(q)+4 M\left(q^{2}\right)\right)+3\left(q \frac{\mathrm{d} L(q)}{\mathrm{d} q}+2 q \frac{\mathrm{d} L\left(q^{2}\right)}{\mathrm{d} q}\right)
$$


and

$$
L(q) L\left(q^{4}\right)=\frac{1}{20}\left(M(q)+3 M\left(q^{2}\right)+16 M\left(q^{4}\right)\right)+\frac{1}{2}\left(3 q \frac{\mathrm{d} L(q)}{\mathrm{d} q}+12 q \frac{\mathrm{d} L\left(q^{4}\right)}{\mathrm{d} q}\right)
$$

(see Theorem 5.1), from which we deduce the arithmetic identities

$$
\begin{aligned}
\sum_{m<n / 2} \sigma(m) \sigma(n-2 m)=\frac{1}{24}\left\{2 \sigma_{3}(n)+8 \sigma_{3}\left(\frac{1}{2} n\right)+\sigma(n)\right. & +\sigma\left(\frac{1}{2} n\right) \\
& \left.-3 n \sigma(n)-6 n \sigma\left(\frac{1}{2} n\right)\right\}
\end{aligned}
$$

and

$$
\begin{aligned}
\sum_{m<n / 4} \sigma(m) \sigma(n-4 m)=\frac{1}{48}\left\{\sigma_{3}(n)+3 \sigma_{3}\left(\frac{1}{2} n\right)\right. & +16 \sigma_{3}\left(\frac{1}{4} n\right)+2 \sigma(n) \\
& \left.+2 \sigma\left(\frac{1}{4} n\right)-3 n \sigma(n)-12 n \sigma\left(\frac{1}{4} n\right)\right\}
\end{aligned}
$$

(see Theorem 5.2), which are due to Melfi $[\mathbf{5}, \mathbf{6}]$ for odd positive integers $n$ and to Huard and co-workers [4] for all positive integers $n$.

\section{Formulae for $L_{r, 4}(q), M_{r, 4}(q)$ and $N_{r, 4}(q)$}

Let $q$ be a real number satisfying

$$
0<q<1
$$

Then

$$
0<-\log q<\infty
$$

The derivative $y^{\prime}$ of the function

$$
y=y(x)=\frac{\pi_{2} F_{1}\left(\frac{1}{2}, \frac{1}{2} ; 1 ; 1-x\right)}{{ }_{2} F_{1}\left(\frac{1}{2}, \frac{1}{2} ; 1 ; x\right)}
$$

is given by

$$
y^{\prime}=-\frac{x^{-1}(1-x)^{-1}}{\left\{{ }_{2} F_{1}\left(\frac{1}{2}, \frac{1}{2} ; 1 ; x\right)\right\}^{2}}
$$

(see, for example, Berndt [1, p. 87]). For $0<x<1$ we have

$$
{ }_{2} F_{1}\left(\frac{1}{2}, \frac{1}{2} ; 1 ; x\right)=\sum_{n=0}^{\infty} \frac{1}{2^{4 n}}\left(\begin{array}{c}
2 n \\
n
\end{array}\right)^{2} x^{n}>0,
$$

so that by (2.4) and (2.5) we see that

$$
y^{\prime}<0 \text { for } 0<x<1 .
$$

By (2.6) $y$ is a strictly decreasing function of $x$ for $0<x<1$. As $y(0)=\infty$ and $y(1)=0$, the function $y$ decreases from $\infty$ to 0 as $x$ increases from 0 to 1 . Hence there exists a unique value of $x$ between 0 and 1 such that

$$
y=\frac{\pi_{2} F_{1}\left(\frac{1}{2}, \frac{1}{2} ; 1 ; 1-x\right)}{{ }_{2} F_{1}\left(\frac{1}{2}, \frac{1}{2} ; 1 ; x\right)}=-\log q .
$$


Therefore,

$$
q=\exp (-y)=\exp \left(-\frac{\pi_{2} F_{1}\left(\frac{1}{2}, \frac{1}{2} ; 1 ; 1-x\right)}{{ }_{2} F_{1}\left(\frac{1}{2}, \frac{1}{2} ; 1 ; x\right)}\right)
$$

We also set

$$
w={ }_{2} F_{1}\left(\frac{1}{2}, \frac{1}{2} ; 1 ; x\right) .
$$

Ramanujan gave in his notebooks [8] the following formulae for $L(q), M(q)$ and $N(q)$ :

$$
\begin{aligned}
L(q) & =(1-5 x) w^{2}+12 x(1-x) w \frac{\mathrm{d} w}{\mathrm{~d} x}, \\
M(q) & =\left(1+14 x+x^{2}\right) w^{4}, \\
N(q) & =(1+x)\left(1-34 x+x^{2}\right) w^{6} .
\end{aligned}
$$

Formulae (2.10)-(2.12) are proved in [2, pp. 127, 129].

It is shown in Berndt [2, p. 125] that if

$$
\Omega(x, q, w)=0
$$

is a relationship between $x, q$ and $w$-where $q$ satisfies (2.1), $x$ is given in terms of $q$ by (2.7) (or (2.8)) and $w$ is given by (2.9) - then the relationship

$$
\Omega\left(\left(\frac{1-\sqrt{1-x}}{1+\sqrt{1-x}}\right)^{2}, q^{2}, \frac{1}{2} w(1+\sqrt{1-x})\right)=0
$$

also holds. The second formula is said to be obtained from the first formula by duplication since $q$ is changed to $q^{2}$.

Applying the process of duplication to (2.10)-(2.12), we obtain

$$
\begin{aligned}
L\left(q^{2}\right) & =(1-2 x) w^{2}+6 x(1-x) w \frac{\mathrm{d} w}{\mathrm{~d} x}, \\
M\left(q^{2}\right) & =\left(1-x+x^{2}\right) w^{4}, \\
N\left(q^{2}\right) & =(1+x)\left(1-\frac{1}{2} x\right)(1-2 x) w^{6}
\end{aligned}
$$

(see [2, pp. 126, 127]). Applying duplication to (2.15)-(2.17), we obtain

$$
\begin{aligned}
L\left(q^{4}\right) & =\left(1-\frac{5}{4} x\right) w^{2}+3 x(1-x) w \frac{\mathrm{d} w}{\mathrm{~d} x}, \\
M\left(q^{4}\right) & =\left(1-x+\frac{1}{16} x^{2}\right) w^{4}, \\
N\left(q^{4}\right) & =\left(1-\frac{1}{2} x\right)\left(1-x-\frac{1}{32} x^{2}\right) w^{6}
\end{aligned}
$$

(see $[\mathbf{2}$, pp. 126, 127]). Again by duplication from $(2.18)-(2.20)$, we have with $g=$ $(1-x)^{1 / 4}$

$$
\begin{aligned}
L\left(q^{8}\right) & =\frac{1}{16}\left(-1+6 g^{2}+11 g^{4}\right) w^{2}+\frac{3}{2} g^{4}\left(1-g^{4}\right) w \frac{\mathrm{d} w}{\mathrm{~d} x}, \\
M\left(q^{8}\right) & =\frac{1}{256}\left(1+60 g^{2}+134 g^{4}+60 g^{6}+g^{8}\right) w^{4}, \\
N\left(q^{8}\right) & =-\frac{1}{4096}\left(1+6 g^{2}+g^{4}\right)\left(1-132 g^{2}-250 g^{4}-132 g^{6}+g^{8}\right) w^{6} .
\end{aligned}
$$


Berndt [2, p. 126] has also described the process of obtaining a new formula from (2.13) by changing the sign of $q$. If (2.13) holds, then the formula

$$
\Omega\left(\frac{x}{x-1},-q, w \sqrt{1-x}\right)=0
$$

also holds. This result is attributed to Jacobi by Berndt [2, p. 126].

Applying Jacobi's change-of-sign procedure to (2.10)-(2.12), we obtain

$$
\begin{aligned}
L(-q) & =(1-2 x) w^{2}+12 x(1-x) w \frac{\mathrm{d} w}{\mathrm{~d} x} \\
M(-q) & =\left(1-16 x+16 x^{2}\right) w^{4} \\
N(-q) & =(1-2 x)\left(1+32 x-32 x^{2}\right) w^{6} .
\end{aligned}
$$

In Cheng [3, pp. 195-208] the process of obtaining a new formula from (2.13) by rotation, that is, $q \rightarrow \mathrm{i} q$, is given and proved. It is shown that if (2.13) holds, then so does the formula

$$
\Omega\left(\frac{-8 \mathrm{i}(1-x)^{1 / 4}(1-\sqrt{1-x})}{\left(1-\mathrm{i}(1-x)^{1 / 4}\right)^{4}}, \mathrm{i} q, \mathrm{i} \frac{1}{2} w\left(1-\mathrm{i}(1-x)^{1 / 4}\right)^{2}\right)=0 .
$$

Applying this process to $(2.10)-(2.12)$, we obtain with $g=(1-x)^{1 / 4}$

$$
\begin{aligned}
& L(\mathrm{i} q)=-\frac{1}{4}\left(1+12 \mathrm{i} g+18 g^{2}-12 \mathrm{i} g^{3}-23 g^{4}\right) w^{2}+12 g^{4}\left(1-g^{4}\right) w \frac{\mathrm{d} w}{\mathrm{~d} x}, \\
& M(\mathrm{i} q)=\frac{1}{16}(1- \\
& \begin{aligned}
N(\mathrm{i} q)=\frac{1}{64}(1- & \left.12 \mathrm{i} g-540 g^{2}+840 \mathrm{i} g^{3}+1094 g^{4}-840 \mathrm{i} g^{5}-540 g^{6}+120 \mathrm{i} g^{7}+g^{8}\right) w^{4} \\
\quad & \left(-1-264 \mathrm{i} g-996 g^{3}+g^{4}\right) \\
& +1848 \mathrm{i} g^{3}+1978 g^{4} \\
& \left.-1848 \mathrm{i} g^{5}-996 g^{6}+264 \mathrm{i} g^{7}-g^{8}\right) w^{6}
\end{aligned}
\end{aligned}
$$

Next, by applying Jacobi's change-of-sign procedure to (2.29)-(2.31), we deduce

$$
\begin{aligned}
& L(-\mathrm{i} q)=-\frac{1}{4}\left(1-12 \mathrm{i} g+18 g^{2}+12 \mathrm{i} g^{3}-23 g^{4}\right) w^{2}+12 g^{4}\left(1-g^{4}\right) w \frac{\mathrm{d} w}{\mathrm{~d} x}, \\
& M(-\mathrm{i} q)=\frac{1}{16}\left(1+120 \mathrm{i} g-540 g^{2}-840 \mathrm{i} g^{3}+1094 g^{4}+840 \mathrm{i} g^{5}-540 g^{6}-120 \mathrm{i} g^{7}+g^{8}\right) w^{4} \\
& N(-\mathrm{i} q)=\frac{1}{64}(1+ \\
& \left.\quad 12 \mathrm{i} g-6 g^{2}-12 \mathrm{i} g^{3}+g^{4}\right) \\
& \times\left(-1+264 \mathrm{i} g-996 g^{2}-1848 \mathrm{i} g^{3}+1978 g^{4}\right. \\
& \left.+1848 \mathrm{i} g^{5}-996 g^{6}-264 \mathrm{i} g^{7}-g^{8}\right) w^{6} .
\end{aligned}
$$

A simple calculation shows that

$$
\begin{aligned}
& L_{0,4}(q)=\frac{1}{96}(4-L(q)-L(-q)-L(\mathrm{i} q)-L(-\mathrm{i} q)), \\
& L_{1,4}(q)=\frac{1}{96}(-L(q)+L(-q)+\mathrm{i} L(\mathrm{i} q)-\mathrm{i} L(-\mathrm{i} q)),
\end{aligned}
$$




$$
\begin{aligned}
L_{2,4}(q) & =\frac{1}{96}(-L(q)-L(-q)+L(\mathrm{i} q)+L(-\mathrm{i} q)), \\
L_{3,4}(q) & =\frac{1}{96}(-L(q)+L(-q)-\mathrm{i} L(\mathrm{i} q)+\mathrm{i} L(-\mathrm{i} q)), \\
M_{0,4}(q) & =\frac{1}{960}(-4+M(q)+M(-q)+M(\mathrm{i} q)+M(-\mathrm{i} q)), \\
M_{1,4}(q) & =\frac{1}{960}(M(q)-M(-q)-\mathrm{i} M(\mathrm{i} q)+\mathrm{i} M(-\mathrm{i} q)), \\
M_{2,4}(q) & =\frac{1}{960}(M(q)+M(-q)-M(\mathrm{i} q)-M(-\mathrm{i} q)), \\
M_{3,4}(q) & =\frac{1}{960}(M(q)-M(-q)+\mathrm{i} M(\mathrm{i} q)-\mathrm{i} M(-\mathrm{i} q)), \\
N_{0,4}(q) & =\frac{1}{2016}(4-N(q)-N(-q)-N(\mathrm{i} q)-N(-\mathrm{i} q)), \\
N_{1,4}(q) & =\frac{1}{2016}(-N(q)+N(-q)+\mathrm{i} N(\mathrm{i} q)-\mathrm{i} N(-\mathrm{i} q)), \\
N_{2,4}(q) & =\frac{1}{2016}(-N(q)-N(-q)+N(\mathrm{i} q)+N(-\mathrm{i} q)), \\
N_{3,4}(q) & =\frac{1}{2016}(-N(q)+N(-q)-\mathrm{i} N(\mathrm{i} q)+\mathrm{i} N(-\mathrm{i} q)) .
\end{aligned}
$$

Using (2.10)-(2.12), (2.25)-(2.27), (2.29)-(2.34) in (2.35)-(2.46), we obtain the following theorem.

\section{Theorem 2.1.}

$$
\begin{aligned}
& L_{0,4}(q)=\frac{1}{192}\left(8+\left(11+18 g^{2}-37 g^{4}\right) w^{2}-96 g^{4}\left(1-g^{4}\right) w \frac{\mathrm{d} w}{\mathrm{~d} x}\right), \\
& L_{1,4}(q)=\frac{1}{32}(1-g)(1+g)^{3} w^{2}, \\
& L_{2,4}(q)=\frac{3}{64}(1-g)^{2}(1+g)^{2} w^{2}, \\
& L_{3,4}(q)=\frac{1}{32}(1-g)^{3}(1+g) w^{2}, \\
& M_{0,4}(q)=\frac{1}{7680}\left(-32+\left(137-540 g^{2}+838 g^{4}-540 g^{6}+137 g^{8}\right) w^{4}\right) \text {, } \\
& M_{1,4}(q)=\frac{1}{64}(1-g)(1+g)^{3}\left(1-3 g+6 g^{2}-3 g^{3}+g^{4}\right) w^{4}, \\
& M_{2,4}(q)=\frac{9}{512}(1-g)^{2}(1+g)^{2}\left(1+6 g^{2}+g^{4}\right) w^{4}, \\
& M_{3,4}(q)=\frac{1}{64}(1-g)^{3}(1+g)\left(1+3 g+6 g^{2}+3 g^{3}+g^{4}\right) w^{4}, \\
& N_{0,4}(q)=\frac{1}{64512}\left(128+\left(1+6 g^{2}+g^{4}\right)\left(2081-8328 g^{2}+12478 g^{4}-8328 g^{6}+2081 g^{8}\right) w^{6}\right) \text {, } \\
& N_{1,4}(q)=\frac{1}{256}(1-g)(1+g)^{3} \\
& \times\left(8-15 g+30 g^{2}-105 g^{3}+172 g^{4}-105 g^{5}+30 g^{6}-15 g^{7}+8 g^{8}\right) w^{6},
\end{aligned}
$$

$$
\begin{aligned}
& N_{2,4}(q)=\frac{33}{1024}(1-g)^{2}(1+g)^{2}\left(1+2 g+2 g^{2}-2 g^{3}+g^{4}\right)\left(1-2 g+2 g^{2}+2 g^{3}+g^{4}\right) w^{6} \\
& N_{3,4}(q)=\frac{1}{256}(1-g)^{3}(1+g) \\
& \quad \quad \times\left(8+15 g+30 g^{2}+105 g^{3}+172 g^{4}+105 g^{5}+30 g^{6}+15 g^{7}+8 g^{8}\right) w^{6}
\end{aligned}
$$

where $g=(1-x)^{1 / 4}$. 
We conclude this section by noting a few results which will be used in $\S \S 3$ and 5 .

The function $w={ }_{2} F_{1}\left(\frac{1}{2}, \frac{1}{2} ; 1 ; x\right)$ satisfies the differential equation

$$
x(1-x) \frac{\mathrm{d}^{2} w}{\mathrm{~d} x^{2}}+(1-2 x) \frac{\mathrm{d} w}{\mathrm{~d} x}-\frac{1}{4} w=0
$$

so that

$$
\frac{\mathrm{d}^{2} w}{\mathrm{~d} x^{2}}=\frac{w}{4 x(1-x)}-\frac{(1-2 x)}{x(1-x)} \frac{\mathrm{d} w}{\mathrm{~d} x} .
$$

By (2.4), (2.7) and (2.9), we have

$$
\frac{1}{q} \frac{\mathrm{d} q}{\mathrm{~d} x}=-\frac{\mathrm{d} y}{\mathrm{~d} x}=\frac{1}{x(1-x) w^{2}}
$$

so that

$$
\frac{\mathrm{d} q}{\mathrm{~d} x}=\frac{q}{x(1-x) w^{2}}
$$

From (2.10), (2.59) and (2.60), we obtain

$$
\begin{aligned}
& \frac{\mathrm{d} L(q)}{\mathrm{d} q} \\
& =\frac{\mathrm{d} L(q)}{\mathrm{d} x} / \frac{\mathrm{d} q}{\mathrm{~d} x} \\
& =\frac{\mathrm{d}}{\mathrm{d} x}\left((1-5 x) w^{2}+12 x(1-x) w \frac{\mathrm{d} w}{\mathrm{~d} x}\right) / \frac{q}{x(1-x) w^{2}} \\
& =\left(-5 w^{2}+(14-34 x) w \frac{\mathrm{d} w}{\mathrm{~d} x}+12 x(1-x)\left(\frac{\mathrm{d} w}{\mathrm{~d} x}\right)^{2}+12 x(1-x) w \frac{\mathrm{d}^{2} w}{\mathrm{~d} x^{2}}\right) / \frac{q}{x(1-x) w^{2}} \\
& =\left(-2 w^{2}+(2-10 x) w \frac{\mathrm{d} w}{\mathrm{~d} x}+12 x(1-x)\left(\frac{\mathrm{d} w}{\mathrm{~d} x}\right)^{2}\right) / \frac{q}{x(1-x) w^{2}}
\end{aligned}
$$

so that

$$
q \frac{\mathrm{d} L(q)}{\mathrm{d} q}=-2 x(1-x) w^{4}+2 x(1-x)(1-5 x) w^{3} \frac{\mathrm{d} w}{\mathrm{~d} x}+12 x^{2}(1-x)^{2} w^{2}\left(\frac{\mathrm{d} w}{\mathrm{~d} x}\right)^{2} .
$$

Similarly from (2.15), (2.18), (2.21), (2.59) and (2.60), we obtain

$$
\begin{aligned}
& q \frac{\mathrm{d} L\left(q^{2}\right)}{\mathrm{d} q}=-\frac{1}{2} x(1-x) w^{4}+2 x(1-x)(1-2 x) w^{3} \frac{\mathrm{d} w}{\mathrm{~d} x}+6 x^{2}(1-x)^{2} w^{2}\left(\frac{\mathrm{d} w}{\mathrm{~d} x}\right)^{2}, \\
& q \frac{\mathrm{d} L\left(q^{4}\right)}{\mathrm{d} q}=-\frac{1}{2} g^{4}\left(1-g^{4}\right) w^{4}+g^{4}\left(1-g^{4}\right)\left(-\frac{1}{2}+\frac{5}{2} g^{4}\right) w^{3} \frac{\mathrm{d} w}{\mathrm{~d} x}+3 g^{8}\left(1-g^{4}\right)^{2} w^{2}\left(\frac{\mathrm{d} w}{\mathrm{~d} x}\right)^{2}
\end{aligned}
$$

and

$$
\begin{aligned}
q \frac{\mathrm{d} L\left(q^{8}\right)}{\mathrm{d} q}=-\frac{1}{16}\left(1-g^{4}\right)\left(3 g^{2}+5 g^{4}\right) w^{4}-\frac{1}{8} g^{4}\left(1-g^{4}\right) & \left(1-6 g^{2}-11 g^{4}\right) w^{3} \frac{\mathrm{d} w}{\mathrm{~d} x} \\
& +\frac{3}{2} g^{8}\left(1-g^{4}\right)^{2} w^{2}\left(\frac{\mathrm{d} w}{\mathrm{~d} x}\right)^{2}
\end{aligned}
$$


In a similar manner we find

$$
\begin{aligned}
q \frac{\mathrm{d} M(q)}{\mathrm{d} q}= & 4 x(1-x)\left(1+14 x+x^{2}\right) w^{5} \frac{\mathrm{d} w}{\mathrm{~d} x}+2 x(1-x)(7+x) w^{6}, \\
q \frac{\mathrm{d} M\left(q^{2}\right)}{\mathrm{d} q}= & -x(1-x)(1-2 x) w^{6}+4 x(1-x)\left(1-x+x^{2}\right) w^{5} \frac{\mathrm{d} w}{\mathrm{~d} x} \\
q \frac{\mathrm{d} M\left(q^{4}\right)}{\mathrm{d} q}= & -\frac{1}{8} x(1-x)(8-x) w^{6}+\frac{1}{4} x(1-x)\left(16-16 x+x^{2}\right) w^{5} \frac{\mathrm{d} w}{\mathrm{~d} x}, \\
q \frac{\mathrm{d} M\left(q^{8}\right)}{\mathrm{d} q}=\frac{1}{256}\left(1-g^{4}\right)( & \left.-30 g^{2}-134 g^{4}-90 g^{6}-2 g^{8}\right) w^{6} \\
& +\frac{1}{64} g^{4}\left(1-g^{4}\right)\left(1+60 g^{2}+134 g^{4}+60 g^{6}+g^{8}\right) w^{5} \frac{\mathrm{d} w}{\mathrm{~d} x} .
\end{aligned}
$$

\section{Products $L_{r, 4}(q) L_{s, 4}(q)$ and $L_{r, 4}(q) M_{s, 4}(q)$}

Using the formulae given in $\S 2$, a MAPLE program was run to determine which of the 10 products $L_{r, 4}(q) L_{s, 4}(q)(0 \leqslant r \leqslant s \leqslant 3)$ can be expressed as a linear combination of

$$
L(q), L\left(q^{2}\right), L\left(q^{4}\right), L\left(q^{8}\right), M(q), M\left(q^{2}\right), M\left(q^{4}\right), M\left(q^{8}\right)
$$

and the derivatives of

$$
L(q), L\left(q^{2}\right), L\left(q^{4}\right) \text { and } L\left(q^{8}\right),
$$

and which of the 10 products $L_{r, 4}(q) M_{s, 4}(q)(0 \leqslant r \leqslant s \leqslant 3)$ can be expressed as a linear combination of

$L(q), L\left(q^{2}\right), L\left(q^{4}\right), L\left(q^{8}\right), M(q), M\left(q^{2}\right), M\left(q^{4}\right), M\left(q^{8}\right), N(q), N\left(q^{2}\right), N\left(q^{4}\right), N\left(q^{8}\right)$ and the derivatives of

$$
L(q), L\left(q^{2}\right), L\left(q^{4}\right), L\left(q^{8}\right), M(q), M\left(q^{2}\right), M\left(q^{4}\right) \text { and } M\left(q^{8}\right) .
$$

Five formulae were found.

Theorem 3.1.

$$
\begin{aligned}
L_{0,4}^{2}(q)=\frac{1}{576}- & \frac{1}{288}\left(7 L\left(q^{4}\right)-6 L\left(q^{8}\right)\right) \\
& +\frac{1}{2880}\left(161 M\left(q^{4}\right)-156 M\left(q^{8}\right)\right)+\frac{1}{48}\left(7 q \frac{\mathrm{d} L\left(q^{4}\right)}{\mathrm{d} q}-6 q \frac{\mathrm{d} L\left(q^{8}\right)}{\mathrm{d} q}\right), \\
L_{2,4}^{2}(q)=\frac{3}{80}\left(M\left(q^{4}\right)-M\left(q^{8}\right)\right), & \\
L_{0,4}(q) L_{2,4}(q)=-\frac{1}{192}( & \left.L\left(q^{2}\right)-3 L\left(q^{4}\right)+2 L\left(q^{8}\right)\right) \\
& +\frac{1}{1920}\left(11 M\left(q^{2}\right)-99 M\left(q^{4}\right)+88 M\left(q^{8}\right)\right) \\
& +\frac{1}{32}\left(q \frac{\mathrm{d} L\left(q^{2}\right)}{\mathrm{d} q}-3 q \frac{\mathrm{d} L\left(q^{4}\right)}{\mathrm{d} q}+2 q \frac{\mathrm{d} L\left(q^{8}\right)}{\mathrm{d} q}\right), \\
L_{1,4}(q) L_{3,4}(q)=\frac{1}{60}\left(M\left(q^{4}\right)-M\left(q^{8}\right)\right), & \\
L_{2,4}(q) M_{2,4}(q)=-\frac{3}{56}( & \left.N\left(q^{4}\right)-N\left(q^{8}\right)\right) .
\end{aligned}
$$


We just give the proof of (3.1).

Proof of (3.1). By (2.18) and (2.21) we have

$$
7 L\left(q^{4}\right)-6 L\left(q^{8}\right)=-\frac{1}{8}\left(11+18 g^{2}-37 g^{4}\right) w^{2}+12 g^{4}\left(1-g^{4}\right) w \frac{\mathrm{d} w}{\mathrm{~d} x},
$$

and from (2.19) and (2.22)

$$
161 M\left(q^{4}\right)-156 M\left(q^{8}\right)=\frac{1}{64}\left(605-2340 g^{2}+3790 g^{4}-2340 g^{6}+605 g^{8}\right) w^{4},
$$

and from (2.63) and (2.64)

$$
\begin{aligned}
7 q \frac{\mathrm{d} L\left(q^{4}\right)}{\mathrm{d} q}-6 q \frac{\mathrm{d} L\left(q^{8}\right)}{\mathrm{d} q} & \\
=\frac{1}{8}\left(9 g^{2}-13 g^{4}\right)\left(1-g^{4}\right) w^{4}-\frac{1}{8} g^{4}\left(1-g^{4}\right)(22 & \left.+36 g^{2}-74 g^{4}\right) w^{3} \frac{\mathrm{d} w}{\mathrm{~d} x} \\
& +12 g^{8}\left(1-g^{4}\right)^{2} w^{2}\left(\frac{\mathrm{d} w}{\mathrm{~d} x}\right)^{2} .
\end{aligned}
$$

Finally,

$$
\begin{aligned}
\frac{1}{576}-\frac{1}{288}( & \left.7 L\left(q^{4}\right)-6 L\left(q^{8}\right)\right)+\frac{1}{2880}\left(161 M\left(q^{4}\right)-156 M\left(q^{8}\right)\right) \\
+\frac{1}{48}( & \left.7 q \frac{\mathrm{d} L\left(q^{4}\right)}{\mathrm{d} q}-6 q \frac{\mathrm{d} L\left(q^{8}\right)}{\mathrm{d} q}\right) \\
= & \frac{1}{576}-\frac{1}{288}\left(-\frac{1}{8}\left(11+18 g^{2}-37 g^{4}\right) w^{2}+12 g^{4}\left(1-g^{4}\right) w \frac{\mathrm{d} w}{\mathrm{~d} x}\right) \\
& +\frac{1}{2880}\left(\frac{1}{64}\right)\left(605-2340 g^{2}+3790 g^{4}-2340 g^{6}+605 g^{8}\right) w^{4} \\
& +\frac{1}{48}\left(\frac{1}{8}\right)\left(9 g^{2}-13 g^{4}\right)\left(1-g^{4}\right) w^{4} \\
& \quad-\frac{1}{48}\left(\frac{1}{8}\right) g^{4}\left(1-g^{4}\right)\left(22+36 g^{2}-74 g^{4}\right) w^{3} \frac{\mathrm{d} w}{\mathrm{~d} x}+\frac{1}{48} 12 g^{8}\left(1-g^{4}\right)^{2} w^{2}\left(\frac{\mathrm{d} w}{\mathrm{~d} x}\right)^{2} \\
= & \frac{1}{36864}\left(8+\left(11+18 g^{2}-37 g^{4}\right) w^{2}-96 g^{4}\left(1-g^{4}\right) w \frac{\mathrm{d} w}{\mathrm{~d} x}\right)^{2} \\
= & L_{0,4}(q)^{2}
\end{aligned}
$$

by $(2.47)$.

This completes the proof of (3.1). The remaining formulae can be proved similarly.

\section{Arithmetic identities}

Equating the coefficients of $q^{n}$ on both sides of the five formulae in Theorem 3.1, we obtain the following theorem. 
Theorem 4.1.

$$
\begin{aligned}
\sum_{m<n} \sigma(4 m) \sigma(4 n-4 m) & =\frac{1}{12}\left\{161 \sigma_{3}(n)-156 \sigma_{3}\left(\frac{1}{2} n\right)\right. \\
& \left.+(1-24 n)\left(7 \sigma(n)-6 \sigma\left(\frac{1}{2} n\right)\right)\right\} \\
\sum_{m \leqslant n} \sigma(4 m-2) \sigma(4 n-(4 m-2)) & =9 \sigma_{3}(n)-9 \sigma_{3}\left(\frac{1}{2} n\right), \\
\sum_{m<n} \sigma(4 m) \sigma(4 n-2-4 m) & =\frac{1}{8}\left\{11 \sigma_{3}(2 n-1)+(13-24 n) \sigma(2 n-1)\right\}, \\
\sum_{m \leqslant n} \sigma(4 m-3) \sigma(4 n-(4 m-3)) & =4 \sigma_{3}(n)-4 \sigma_{3}\left(\frac{1}{2} n\right), \\
\sum_{m \leqslant n} \sigma_{3}(4 m-2) \sigma(4 n-(4 m-2)) & =27 \sigma_{5}(n)-27 \sigma_{5}\left(\frac{1}{2} n\right) .
\end{aligned}
$$

Let $a$ and $b$ be integers satisfying $b \geqslant 1$ and $0 \leqslant a \leqslant b-1$. Set

$$
S(a, b)=\sum_{\substack{m=1 \\ m \equiv a(\bmod b)}}^{n-1} \sigma(m) \sigma(n-m) .
$$

Huard and co-workers $[\mathbf{4}, \S 5]$ have given results for $S(a, b)$ for $b=1,2,3$ and 4 .

Formula (4.4) gives the value of $S(1,4)$ for $n \equiv 0(\bmod 4)$ [4, Theorem 9]. Formula $(4.3)$ gives the value of $S(0,4)$ for $n \equiv 2(\bmod 4)[4$, Theorem 9]. Formula $(4.2)$ gives the value of $S(2,4)$ for $n \equiv 0(\bmod 4)$. Formula $(4.1)$ gives the value of $S(0,4)$ for $n \equiv 0(\bmod 4)$. The latter two formulae extend the result given in $[4$, Theorem 9].

\section{Further relations}

It is possible to derive many other relations similar to those in Theorems 3.1 and 4.1 . We refer the reader to Cheng [3] for details. We just give two other identities.

Theorem 5.1.

$$
\begin{aligned}
& L(q) L\left(q^{2}\right)=\frac{1}{5}\left(M(q)+4 M\left(q^{2}\right)\right)+3\left(q \frac{\mathrm{d} L(q)}{\mathrm{d} q}+2 q \frac{\mathrm{d} L\left(q^{2}\right)}{\mathrm{d} q}\right), \\
& L(q) L\left(q^{4}\right)=\frac{1}{20}\left(M(q)+3 M\left(q^{2}\right)+16 M\left(q^{4}\right)\right)+\frac{1}{2}\left(3 q \frac{\mathrm{d} L(q)}{\mathrm{d} q}+12 q \frac{\mathrm{d} L\left(q^{4}\right)}{\mathrm{d} q}\right) .
\end{aligned}
$$

Proof. First we prove (5.1). By (2.11) and (2.16) we have

$$
M(q)+4 M\left(q^{2}\right)=5\left(1+2 x+x^{2}\right) w^{4}
$$

and from (2.61) and (2.62)

$$
\begin{aligned}
& q \frac{\mathrm{d} L(q)}{\mathrm{d} q}+2 q \frac{\mathrm{d} L\left(q^{2}\right)}{\mathrm{d} q} \\
& \quad=-3 x(1-x) w^{4}+6 x(1-x)(1-3 x) w^{3} \frac{\mathrm{d} w}{\mathrm{~d} x}+24 x^{2}(1-x)^{2} w^{2}\left(\frac{\mathrm{d} w}{\mathrm{~d} x}\right)^{2} .
\end{aligned}
$$


Hence

$$
\begin{aligned}
\frac{1}{5}(M(q) & \left.+4 M\left(q^{2}\right)\right)+3\left(q \frac{\mathrm{d} L(q)}{\mathrm{d} q}+2 q \frac{\mathrm{d} L\left(q^{2}\right)}{\mathrm{d} q}\right) \\
= & (1-2 x)(1-5 x) w^{4}+18 x(1-x)(1-3 x) w^{3} \frac{\mathrm{d} w}{\mathrm{~d} x}+72 x^{2}(1-x)^{2} w^{2}\left(\frac{\mathrm{d} w}{\mathrm{~d} x}\right)^{2} \\
= & L(q) L\left(q^{2}\right)
\end{aligned}
$$

by $(2.10)$ and $(2.15)$.

Next we prove (5.2). By (2.11), (2.16) and (2.19) we have

$$
M(q)+3 M\left(q^{2}\right)+16 M\left(q^{4}\right)=5\left(4-x+x^{2}\right) w^{4}
$$

and from (2.61) and (2.63)

$$
\begin{aligned}
3 q \frac{\mathrm{d} L(q)}{\mathrm{d} q}+ & 12 q \frac{\mathrm{d} L\left(q^{4}\right)}{\mathrm{d} q} \\
& =-12 x(1-x) w^{4}+30 x(1-x)(1-2 x) w^{3} \frac{\mathrm{d} w}{\mathrm{~d} x}+72 x^{2}(1-x)^{2} w^{2}\left(\frac{\mathrm{d} w}{\mathrm{~d} x}\right)^{2} .
\end{aligned}
$$

Hence

$$
\begin{aligned}
\frac{1}{20}(M(q) & \left.+3 M\left(q^{2}\right)+16 M\left(q^{4}\right)\right)+\frac{1}{2}\left(3 q \frac{\mathrm{d} L(q)}{\mathrm{d} q}+12 q \frac{\mathrm{d} L\left(q^{4}\right)}{\mathrm{d} q}\right) \\
& =(1-5 x)\left(1-\frac{5}{4} x\right) w^{4}+15 x(1-x)(1-2 x) w^{3} \frac{\mathrm{d} w}{\mathrm{~d} x}+36 x^{2}(1-x)^{2} w^{2}\left(\frac{\mathrm{d} w}{\mathrm{~d} x}\right)^{2} \\
& =L(q) L\left(q^{4}\right)
\end{aligned}
$$

by (2.10) and (2.18). This completes the proof of Theorem 5.1.

Equating the coefficients of $q^{n}$ on both sides of (5.1) and (5.2), we obtain the following theorem.

Theorem 5.2.

$$
\begin{aligned}
& \sum_{m<n / 2} \sigma(m) \sigma(n-2 m) \\
& \quad=\frac{1}{24}\left\{2 \sigma_{3}(n)+8 \sigma_{3}\left(\frac{1}{2} n\right)+\sigma(n)+\sigma\left(\frac{1}{2} n\right)-3 n \sigma(n)-6 n \sigma\left(\frac{1}{2} n\right)\right\} \\
& \sum_{m<n / 4} \sigma(m) \sigma(n-4 m) \\
& \quad=\frac{1}{48}\left\{\sigma_{3}(n)+3 \sigma_{3}\left(\frac{1}{2} n\right)+16 \sigma_{3}\left(\frac{1}{4} n\right)+2 \sigma(n)+2 \sigma\left(\frac{1}{4} n\right)-3 n \sigma(n)-12 n \sigma\left(\frac{1}{4} n\right)\right\} .
\end{aligned}
$$

Formulae (5.3) and (5.4) are due to Melfi $[\mathbf{5}, \mathbf{6}]$ for $n$ odd and to Huard and coworkers [4] for all $n$.

Acknowledgements. The research of K.S.W. was supported by the Natural Sciences and Engineering Research Council of Canada under grant A-7233. 


\section{References}

1. B. C. BeRndt, Ramanujan's notebooks, Part II (Springer, 1989).

2. B. C. Berndt, Ramanujan's notebooks, Part III (Springer, 1991).

3. N. Cheng, Convolution sums involving divisor functions, MSc thesis, Carleton University, Canada (2003).

4. J. G. Huard, Z. M. Ou, B. K. Spearman and K. S. Williams, Elementary evaluation of certain convolution sums involving divisor functions, in Number theory for the Millennium, vol. II (ed. M. A. Bennett et al.), pp. 229-274 (A. K. Peters, Natick, MA, 2002).

5. G. Melfi, Some problems in elementary number theory and modular forms, $\mathrm{PhD}$ thesis, University of Pisa, Italy (1997).

6. G. Melfi, On some modular identities, in Number Theory, Diophantine, Computational and Algebraic Aspects: Proc. Int. Conf., Eger, Hungary, pp. 371-382 (Walter de Grutyer, Berlin, 1998).

7. S. Ramanujan, On certain arithmetical functions, Trans. Camb. Phil. Soc. 22 (1916), 159-184.

8. S. Ramanujan, Notebooks, 2 vols (Tata Institute of Fundamental Research, Bombay, 1957).

9. S. Ramanujan, Collected papers (AMS Chelsea Publishing, Providence, RI, 2000).

10. N.-P. Skoruppa, A quick combinatorial proof of Eisenstein series identities, J. Number Theory 43 (1993), 68-73. 